\title{
Pulsating white dwarfs as astrophysical tools
}

\author{
Denis J. Sullivan \\ School of Chemical 8 Physical Sciences, Victoria University of \\ Wellington, P.O. Box 600, Wellington, New Zealand
}

\begin{abstract}
The study of pulsating white dwarfs is a scientifically productive exercise as their measured pulsation characteristics yield both unique data on their interiors and also shed light on more general astrophysical questions. The modelling process is simplified due to their assumed chemically homogeneous and layered structures. As well as being archaeological remnants of their nuclear burning progenitors, white dwarfs are cosmic laboratories that can provide some observational constraints on theories of matter in regions of phase space that are unattainable in laboratories on Earth. The hotter white dwarfs should feature neutrino production by several unusual weak interaction processes, while their cooler cousins should exhibit core crystallization. White dwarf pulsators are very stable in period (if not always in amplitude) and suitable objects can be used as sensitive orbiting clocks in a search for stellar companions, including those of planetary size. Finally, the ensemble of cooling white dwarfs can be used as an independent cosmic chronometer over very long timescales if the cooling curve is properly calibrated. The pulsators make important contributions in this regard.
\end{abstract}

\section{Introduction}

The current received wisdom of stellar evolution theory is that the great majority of stars will end their lives as slowly cooling white dwarfs. As such and separate from any intrinsic interest, they are an important archaeological record of the prior nuclear burning phases of their progenitors. Consequently, their study provides unique insights into these earlier and more complex evolutionary phases.

In stellar terms, a white dwarf (WD) is a simple object: a hot essentially isothermal core with an overlaying insulating blanket of material exhibiting many of the properties of a "normal" (albeit high gravity) stellar atmosphere. The interior regions are dominated by the properties of a plasma with two separate components. The collection of nuclei is overwhelmingly the source of the object's confining self gravity, and these same nuclei constitute an essentially classical gas which is the source of the thermal energy that fuels the object's luminosity for most of its cooling phase. In stark contrast, the electrons act separately and their quantum behaviour is responsible for the degeneracy pressure resisting the inward pull of gravity deriving from their more massive brethren. The cooler the white dwarf, the more accurate is this simple picture for the electrons, but the nuclei eventually take on some of the properties of a crystalline 
solid. One could say that the electrons and nuclei are locked in struggle of stellar proportions and the end result is a stalemate that cools into observational oblivion.

It is also fruitful to view white dwarfs as cosmic laboratories for studying the properties of material at extremely high temperatures and densities. In the hotter cooling phases of white dwarf evolution, theory predicts that large neutrino fluxes will be produced in the core via the weak interaction. In contrast, it is expected that at the cool end of the evolution of a WD, core crystallization will gradually emerge as a feature due to the ensemble of nuclei seeking the lowest energy state.

We are fortunate that nature has also provided us with a mechanism to probe white dwarf interiors: they are seen to pulsate in several regions of their cooling phases and these pulsations are sensitive probes of the internal physical properties.

\section{The pulsating white dwarfs}

As white dwarfs cool, they become unstable to nonradial gravity mode mechanical pulsation with periods in the range $10^{2}-10^{3} \mathrm{~s}$ (e.g., see Winget 1998 for a review). These buoyancy-driven pulsations are normal mode oscillations of the whole fluid sphere and they occur in several distinct temperature regions. Theory has it that the phenomenon results from changing partial ionization conditions in the outer layers of the star as a result of evolutionary cooling. Therefore it can be surmised that the pulsators are representative of white dwarfs in general. The pulsators are invariably multiperiodic, so the tools of asteroseismology can be usefully invoked to yield structural information. In essence, this procedure uses the detected pulsation spectrum in a star to constrain the parameters of appropriate numerical models, and thereby improve the model parameters and the physical basis of the model.

The very hot pre-white dwarf (DOV) objects pulsate in temperature regimes in excess of $T_{\text {eff }} \sim 80000 \mathrm{~K}$, the helium atmosphere (DBV) white dwarfs pulsate when $T_{\text {eff }} \sim 25000 \mathrm{~K}$, and the hydrogen atmosphere (DAV) objects pulsate in an instability strip $11000 \lesssim T_{\text {eff }} \leqslant 12500 \mathrm{~K}$.

The assumed chemically homogeneous layered structures of white dwarfs that results from a lack of nuclear burning reactions and the gravitational settling of the different chemical species in the strong gravitational fields makes the modelling task relatively simple.

This brief review will be necessarily incomplete, but several interesting recent developments will be discussed.

\section{The hydrogen atmosphere pulsating white dwarfs}

The DA hydrogen atmosphere white dwarfs constitute about $80 \%$ of the known white dwarf population, and it was one of these (HL Tau 76) that was serendipitously discovered to pulsate about 35 years ago. Since then, various surveys have increased the known number of such pulsators to something in excess of 
30. This number has been doubled in the last few years as the result of an observing campaign at McDonald Observatory in Texas (Mukadam et al. 2004).

\subsection{The McDonald Observatory DAV search}

There are a number of important components to this observing campaign. First, a new source of (fainter) white dwarfs has become available from the Sloan Digital Sky Survey (SDSS) and the Hamburg Quasar Survey (HQS). Although these surveys are focussed on extragalactic targets, they also obtain some data on other interesting objects.

Second, survey colour data combined with atmospheric model fitting (Koester, private communication) to SDSS spectra is undertaken in order to identify suitable white dwarf DAV candidates. Improving the efficiency of these preselection procedures has been an important aspect of the campaign (Mukadam et al. 2004), as there are simply too many candidate WDs produced by these surveys (e.g., Harris et al. 2003), and each observed target object uses a minimum of about half an hour of scarce telescope time.

Third, the observing programme utilises a new frame transfer CCD photometer ("Argos", Nather \& Mukadam 2003) that can obtain useful time series photometry of objects as faint as nearly 20th magnitude, when mounted at the prime focus of the McDonald Observatory 2.1-m telescope.

And fourth, an intensive observational campaign to identify actual pulsators has been underway at McDonald since late 2001.

The motivation behind this observing programme is to significantly increase the known sample of DAV pulsators in order to investigate a number of interesting astrophysical problems. Several examples are as follows.

\subsection{Astrophysics with more DAVs}

The hot DAV pulsators (HDAVs) are very stable in both frequency and amplitude, and can thus be thought of as excellent clocks. If such a WD has an orbital companion, its motion around the common centre of mass of the two objects will lead to a periodic variation in the photon arrival times (if the plane of the orbit is suitably aligned); and this will be observable as a change in the observed phase of a sufficiently stable pulsation. This technique can be used to specifically search for WD orbital companions, and planets in particular (Winget et al. 2003). As an example, a Jupiter-mass planet orbiting a $0.6-\mathrm{M}_{\odot} \mathrm{WD}$ at a distance of about $5 \mathrm{AU}$ should be detectable if the pulsator is as stable as the white dwarf (G117-B15A) studied extensively by Kepler et al. (2000).

It is likely that at least some planets should survive the earlier stages in a star's evolution and therefore be present during the WD cooling phase. Even a null result for a statistically significant sample of suitable pulsating WDs would be an interesting measurement, as it would provide constraints on the planetary formation rate (or cast doubt on the planetary survival hypothesis).

Provided the WD cooling rate is properly understood, white dwarfs as a group can be used to infer the age of the local galactic disk where they are found (e.g., Fontaine, Brasard \& Bergeron 2001). This will then lead to useful estimates of the age of our galaxy itself. Having access to a large sample of stable HDAVs with different masses and possibly internal compositions will allow constraints to be placed on period change rates, which in turn provides data on 
cooling rates. Large uncertainties in the ${ }^{12} \mathrm{C}(\alpha, \gamma){ }^{16} \mathrm{O}$ cross section at the low interaction energies appropriate to stellar interiors limit our knowledge of the WD core compositions (e.g., Metcalfe, Salaris \& Winget 2002; Kunz et al. 2002). Even simple constraints on period change rates for the DAVs provides useful data here.

With an increased number of DAVs, it is more likely that a massive pulsator, similar to the object BPM 37093 will be found. This latter object has been extensively studied, including via several Whole Earth Telescope (WET, Nather et al. 1990) runs (e.g., Nitta et al. 1999), in an effort to observationally confirm the theoretically expected partial core crystallisation (e.g., Lamb \& van Horn 1975).

Finally, a larger sample of DAV pulsators will also allow the physics of the WD hydrogen atmosphere instability strip to be scrutinised in more detail. Such questions as the contribution the WD mass (i.e. surface gravity) makes to the location of the instability strip phase space should be answered with more clarity.

\section{The hot white dwarf pulsators}

The hot pulsators come in two rather different flavours. The very hot (strictly, pre-) white dwarfs (DOVs) occur for $T_{\text {eff }} z 80000 \mathrm{~K}$. while the helium atmosphere DBVs occur around $T_{\text {eff }} \sim 25000 \mathrm{~K}$.

It is common to refer to a white dwarf cooling track that includes the 3 flavours of pulsating WDs and implies a common evolutionary heritage. But there are a number of observational facts that cloud this tidy picture. Probably the most significant fact is the so-called DB "gap", whereby there are no observed such objects with $T_{\text {eff }}$ between about 30000 and $45000 \mathrm{~K}$. As one can anticipate, theories have been advanced that explain the "gap" and preserve a common ancestry for the WD classes. Nevertheless, one might say that "the jury is still out" on this issue.

\subsection{The helium atmosphere pulsating white dwarfs}

The usually assumed structure for these objects is a carbon/oxygen core with an overlaying thin atmosphere of helium. This chemically stratified model is the simplest of all the WDs and therefore the easiest to model. Currently, there is a total of 9 pulsating DBs that are known, and the class prototype (GD 358) has been extensively studied, including via 3 separate WET runs (e.g., Kepler et al. 2003).

Extensive matching of models to the observational pulsation data has been undertaken for GD 358, in part because of the relative simplicity of the models and in part because of the extensive nature of the observational data sets. This work has recently led to an independent asteroseismological measurement of the ${ }^{12} \mathrm{C}(\alpha, \gamma){ }^{16} \mathrm{O}$ cross section relevant for the energies in stellar interiors (Metcalfe, Salaris \& Winget 2002), something that is very difficult to achieve in the laboratory (Kunz et al. 2002).

But arguably the most interesting potential outcome of studying the hot DBV white dwarfs is to see them invoked as neutrino detectors. 


\subsection{Neutrinos and white dwarfs}

Irrespective of whether the DO and DB white dwarfs have a common lineage, there is a definite common theoretical feature for these hot objects: their plasma interiors are in a region of matter phase space where it is predicted that neutrinos will be created in large numbers. These neutrino creation processes are different from the nucleon conversion processes in the nuclear reactions occurring in other stars. The weak interaction is still responsible, but it is the generalised form of this interaction at work in the extreme plasma conditions that constitute WD interiors.

Several years after Fermi's point weak interaction theory was generalised in the late 1950s by Feynman \& Gell-Mann (1958) and others, a variety of theoretical papers appeared in the literature suggesting that a number of new weak interaction processes should be important in the very hot dense plasmas likely to be found in certain astrophysical situations (see Fowler \& Hoyle 1964 for an early review). Subsequent work on weak interaction processes ultimately led to the modern unified electro-weak theory of Glashow, Salam \& Weinberg (e.g., Weinberg 1967; Martin \& Shaw 1997), and laboratory accelerator experiments have confirmed all the predictions of this general theory at high energies. However, the key new idea that was important for white dwarf plasmas was part of the initial generalisation of Fermi's theory, namely the suggestion of a direct interaction between the electron and the neutrino without any nucleon involvement.

For white dwarf interiors $\left(\mathrm{T}_{c} \sim 5 \times 10^{7} \mathrm{~K}, \rho_{c} \lesssim 5 \times 10^{6} \mathrm{~g} \mathrm{~cm}^{-3}\right)$, two particular neutrino production mechanisms are significant. First, an electron changing its momentum in the Coulomb field of a nucleus can emit a neutrino anti-neutrino pair (bremsstrahlung neutrinos, Pontecorvo 1959), rather than the usual photon. Second, a photon propagating in a plasma develops an effective rest mass by interacting with the plasma, and this massive photon (a plasmon) can then decay into a neutrino pair and still satisfy the energy and momentum conservation laws. This plasmon neutrino decay mechanism was first discussed by Adams, Ruderman \& Woo (1963) and it turns out to be the dominant form of neutrino production in WD interiors.

In spite of the high density of white dwarf core material, the extremely small cross sections for neutrino interaction with matter means that neutrinos created there will escape unimpeded. Consequently, the energy they extract from the star will add directly to the surface photon flux as a cooling mechanism. The spectacular weakness of the neutrino interactions also means that their production mechanisms are relatively very improbable. However, the gravitationally trapped plasma of a white dwarf interior provides an environment in which the neutrino production and consequent energy loss is competitive with the slow thermal photon leakage via the much cooler surface.

Neutrino contributions have been included in white dwarf model calculations since the $1960 \mathrm{~s}$, beginning with pioneering work at the University of Rochester (e.g., Vila 1966; Kutter \& Savedoff 1969; Lamb \& Van Horn 1975) using neutrino rates published in Beaudet, Petrosian \& Salpeter (1967). Subsequent work has included updated rates (which are not dramatically different) based on the electro-weak theory of Weinberg et al. - see Metcalfe, Nather $\&$ Winget (2000) for a brief description of relevant models. These models all 
demonstrate that the neutrino flux exceeds the photon flux by a significant margin for the very hot pre-white dwarfs.

O'Brien et al. (1998) and O'Brien \& Kawaler (2000) have analysed data (including a WET run) on the DOV object PG $0122+200\left(T_{\text {eff }} \gtrsim 80000 \mathrm{~K}\right)$ and attempted to measure cooling-induced changes in the periods as a means of indirectly detecting evidence of a neutrino flux. One definite conclusion was that more data were required to achieve this aim.

Recent WD modelling investigations (Winget et al. 2003) have shown that the neutrino flux continues to exceed the photon flux down to effective temperatures characteristic of the DBV white dwarfs, provided the WD mass is not too large. The object EC 20058-5234 is the hottest known DBV object and it is likely that neutrino core cooling will play a significant role.

\section{The DBV white dwarf EC 20058-5238}

The object EC 20058-5234 was identified as a helium atmosphere white dwarf by the Edinburgh-Cape survey of faint blue objects, and its classification and pulsation properties were first reported by Koen et al. (1995). Its pulsation spectrum was further investigated during a WET run in 1997 (XCOv15). A paper reporting the results of this WET run is currently in preparation (Sullivan et al. 2004), but it is relevant to note here that the superior WET data set extended the number of detected independent pulsation modes from the 6 obtained by Koen et al. to at least 11.

However, using the set of revealed modes as the basis of an asteroseismological analysis, similar to that performed on the class prototype (GD 358) by Bradley \& Winget (1994) has proven to be difficult. The nearly-uniformlyspaced $\ell=1$ periods in this latter object that were detected in a WET run were the basis for deducing a number of significant astrophysical results. Even though an analysis of subsequent WET runs (Kepler et al. 2003) revealed that the pulsation spectrum of GD 358 is rather complicated and changes with time, the basic results remained unchanged.

In contrast, the pulsation spectrum of EC 20058 does not exhibit an obvious period spacing, has shorter periods and undoubtedly includes at least one $\ell=2$ mode. This scenario is suggested in the discovery work and the analysis of the WET data (Sullivan et al. 2004) does not substantially change this picture. Consequently, some asteroseismological techniques, such as matching an observed uniform period spacing to asymptotic (high radial order) model predictions are not applicable.

It is possible that using the genetic-algorithm technique to explore model parameter space (Metcalfe, Nather \& Winget 2000) will provide useful physical results as have been achieved for GD 358 (Metcalfe, Salaris \& Winget 2002), but the nature of the revealed modes for EC 20058 leads to uniqueness matching problems. However, work in this area is continuing.

A spectroscopic analysis by Beauchamp et al. (1999) has deduced an effective temperature for EC 20058 as high as $28400 \mathrm{~K}$. For such a temperature, the core neutrino flux plays a very significant role in the cooling process. The pulsation spectrum of this object is dominated by two modes with periods near 257 and $281 \mathrm{~s}$ (see Fig. 1), and the author has been regularly monitoring this pul- 


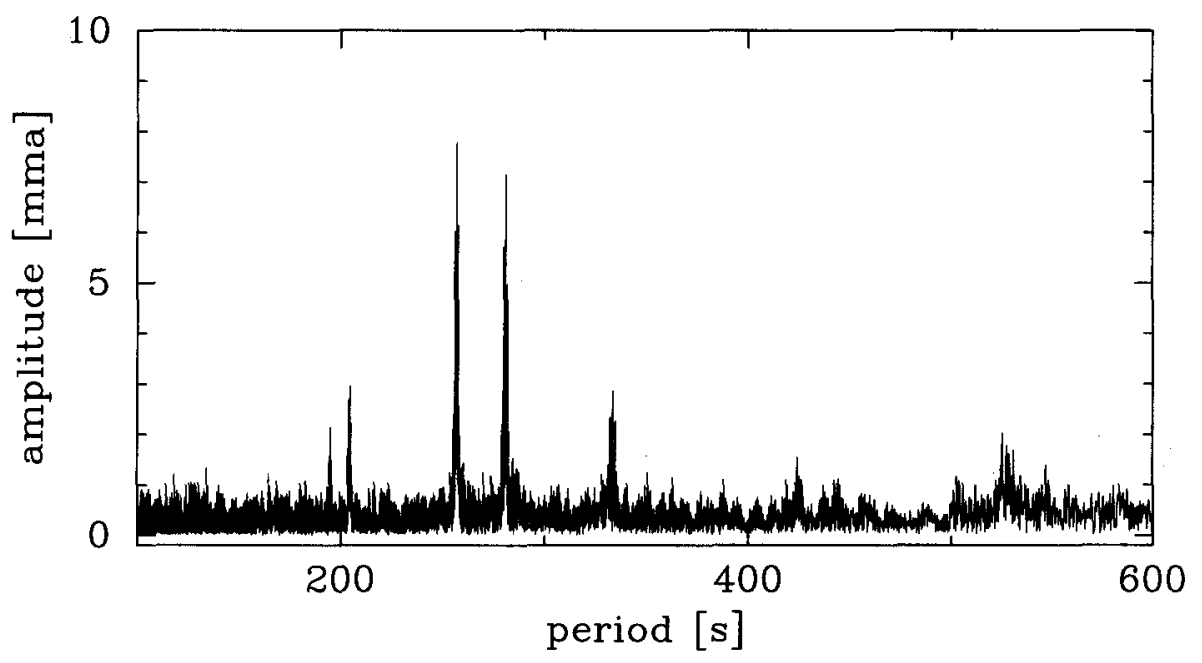

Figure 1. A amplitude spectrum for EC 20058 obtained recently from 4 nights observing at Mt John in 2002 August.

sator at Mt John Observatory (Lake Tekapo, NZ) since the WET run in 1997 (Sullivan 2003). The observing programme has been focussed on investigating the amplitude and frequency stability of the pulsation modes of EC 20058. It is possible that a period increase consistent with the cooling effect of the predicted neutrino cooling flux will be detected in the future. Such a result would be a confirmation of the existence of the underlying neutrino processes and therefore a unique low energy test of the electro-weak theory of lepton interactions.

It is interesting to compare potential neutrino measurements for the two classes of hot white dwarfs. The DBVs are the simplest of all the white dwarfs to model as they are supposed to have only a thin helium atmosphere overlaying a carbon/oxygen core. In addition, their temperatures are sufficiently low that the mechanical structure is effectively decoupled from the thermal structure. On the other hand, the DOVs are more complicated and the thermal and mechanical properties are still interlinked due to the much higher temperatures. Therefore a neutrino "measurement" using a DBV would be more independent of any modelling uncertainties.

Acknowledgments. I would like to thank various members of the University of Texas at Austin white dwarf research group, past and present, including Don Winget, Ed Nather, Anjum Mukadam, Fergal Mullally, Scot Kleinman, Atsuko Nitta and others for various collaborative endeavours. I would also like to express my appreciation to the various members of the WET collaboration I have worked with. 


\section{References}

Adams, J.B., Ruderman, M.A., Woo, C.H. 1963, Phys. Rev., 129, 1383

Beauchamp, A., et al. 1999, ApJ, 516, 887

Beaudet, G., Petrosian, V., Salpeter, E.E. 1967, ApJ, 150, 979

Bradley, P.A., Winget, D.E. 1994, ApJ430, 850

Feynman, R.P., Gell-Mann, M. 1958, Phys. Rev., 109, 193

Fontaine, G., Brasard, P., Bergeron, P. 2001, PASP, 113, 409

Fowler, W.A., Hoyle, F. 1964, ApJS, 9, 201

Harris, H.C., et al. 2003, AJ, 126, 1023

Kepler, S.O., et al. 2000, ApJ, 534, L185

Kepler, S.O., et al. 2003, A\&A, 401, 639

Koen, C., et al. 1995, MNRAS, 277, 913

Kunz, R., Fey, M., Jaeger, M., Mayer, A., Hammer, J.W. 2002, ApJ, 567, 643

Kutter, G.S., Savedoff, M.P. 1969, ApJ, 157, 1021

Lamb, D.Q., Van Horn, H.M. 1975 ApJ, 200, 306

Martin, B.R., Shaw, G. 1997, Particle Physics (Chichester: Wiley)

Metcalfe, T.S., Nather, R.E., Winget, D.E. 2000, ApJ, 545, 974

Metcalfe, T.S., Salaris, M., Winget, D.E. 2002, ApJ, 573, 803

Mukadam, A.S., et al. 2004, ApJ, submitted

Nather, R.E., Mukadam, A.S. 2003, PASP, in press

Nather, R.E. et al. 1990, ApJ, 361, 309

Nitta, A. et al. 1999, in ASP Conf. Ser., Vol. 169, $11^{\text {th }}$ European Workshop on White Dwarfs, eds J.-E. Solheim \& E.G. Meistas (San Francisco: ASP), 144

O'Brien, M.S., Kawaler, S.D. 2000, ApJ, 539, 372

O'Brien, M.S., et al. (the WET collaboration) 1998, ApJ, 495, 458

Pontecorvo, B. 1959, Soviet Phys., J.E.T.P., 9, 1148

Sullivan, D.J. 2003, in White Dwarfs, eds D. de Martino, R. Silvotti, J.-E.

Solheim. R. Kalytis, (Dordrecht: Kluwer), 231

Sullivan, D.J., et al. (the WET collaboration) 2004, in preparation

Vila, S.C. 1966, ApJ, 146, 437

Weinberg, S. 1967, Phys.Rev.Lett, 19, 1264

Winget, D.E. 1998, J. Phys. Condens. Matter, 10, 11247

Winget, D.E., et al. 2003, in ASP Conf. Ser., Vol. 294, Scientific Frontiers in Research on Extrasolar Planets, eds D. Deemimg \& S. Seager (San Francisco: ASP), in press

Winget, D.E., Sullivan, D.J., Metcalf, T.S, Kawaler, S.D., Montgomery, M.H. 2003 ApJ, submitted 


\section{Discussion}

Cassisi: You have discussed the effects of neutrino energy losses on the pulsational properties of WD, but what are the effects of different assumptions on the chemical stratification in the C/O CORE?

Sullivan: The assumed core chemical composition profiles in the WD models do affect the calculated $g$-mode frequencies at the detail level, and you can see some of the specifics in, for example, Metcalfe, Salaris \& Winget (2002, and references therein). However, several general effects are easily summarised. For a given WD mass, an increase in the oxygen content relative to carbon decreases the core heat capacity, which reduces the cooling timescale; and this increases the overall rate of increase with time of the pulsation periods. Also, the additional core cooling due to neutrino energy emission has its major impact on period change rates and has only a small effect on the actual pulsation periods.

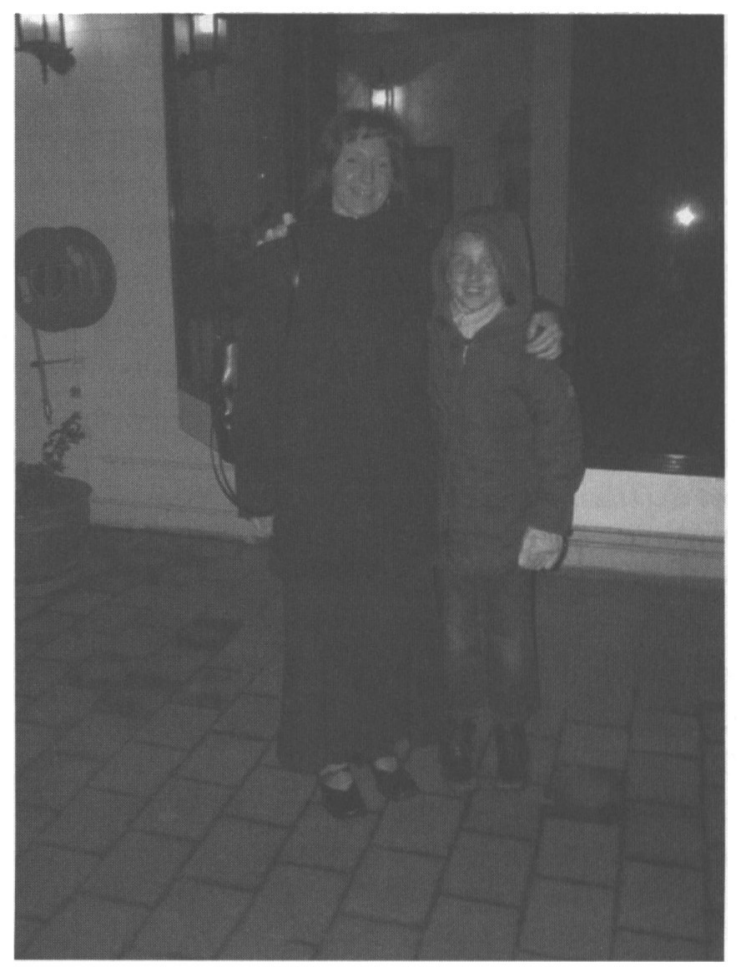

Big and Little Conny Aerts 